\title{
P-0576 - In search of cardiovascular risks reduction in type 2 diabetes: Transcriptional regulation of meal replacement therapy
}

Tay, S.H.(1) (3)*; Billa, N.(1); Goh, Y.M.(2); Hakimah, M.S.(3); Mohd Yusof, B.N.(4); Pung, Y.F.(5); Gew, S.P.(4); Ramachandran, V.(6); Hoo, F.K.(3).

\author{
1 The University of Nottingham Malaysia Campus, School of Pharmacy, Semenyih, Malaysia. \\ 2 University Putra Malaysia, Faculty of Veterinary Medicine, Selangor, Malaysia. \\ 3 University Putra Malaysia, Department of Medicine, Selangor, Malaysia. \\ 4 University Putra Malaysia, Department of Nutrition and Dietetics, Selangor, Malaysia. \\ 5 The University of Nottingham Malaysia Campus, Department of Biomedical Sciences, Semenyih, Malaysia. \\ 6 Malaysian Research Institute on Ageing.
}

\section{BACKGROUND:}

$>$ Lifestyle intervention such as meal replacement therapy (MRT) is the cornerstone in managing diabetes complication such as cardiovascular disease (CVD). However, the underlying transcriptional mechanism of MRT attributed to favorable result in CVD remain elusive. Dysregulation of peroxisome proliferator-activated receptors (PPARs) may delineate the pathogenesis of obesity, T2D, dyslipidemia and CVD individual.

\section{AIM:}

To uncover the transcriptional regulation underlying the improvement of CVD risks among T2D patients induced by MRT

\section{METHODS:}

We randomized obese T2D either to isocaloric (1200kcal) MRT or standard dietary therapy (SDT), for 12 weeks intervention. We genotyped PPARy SNP, Pro12Ala using allele specific real-time polymerase chain reaction (rtPCR). PPARs and their target genes were quantitated with rt-PCR at baseline and $12^{\text {th }}$ weeks of intervention. Multiple linear regression model was used to inspect the meaningful predictive value of underlying molecular markers and pathway to CVD risk factors. All statistical procedures were conducted at $95 \%$ confidence level.

\section{RESULTS and DISCUSSIONS:}

MRT arm had significantly reduced total cholesterol/ high-density lipoprotein cholesterol ratio (TC/HDL-C), a well-known atherogenic index of CVD risks from $(4.82 \pm 0.43$ to $4.43 \pm 0.38, P<0.05)$ and Table 1 depicted the additional CVD risk factors reduced by MRT arm $(\mathrm{P}<0.05)$, regardless of Pro12Ala SNP predisposition

$>$ Clinical phenotypes such as high-sensitivity C-reactive protein (hs-CRP) and visceral fat reduction have demonstrated to predict TC/HDL-C reduction by $42 \%$ and $38 \%$, respectively $(\mathrm{P}<0.05)$

$>$ Transcriptional PPARY activation has shown to explain $50.7 \%$ of the TC/HDL-C reduction in MRT participants $\left(R^{2}=0.507, \beta=-0.71(-0.07-(-0.01), P<0.05)\right.$ Fatty acid oxidation, solute carrier family 25 (carnitine/acylcarnitine translocase), member 20 (SLC25A20) activation could potentially explain how visceral fat contributed to reduction of atherogenic index TC/HDL-C by $\left(R^{2}=0.650, \beta=\right.$ $0.03(-0.05-(-0.01), P<0.05)$

\section{CONCLUSIONS:}

> The transcriptional activation of PPARY and SLC25A20 have displayed to significantly modulate the favorable CVD risk factors improvement by MRT, among T2D individual with obesity.

\section{Table 1: CVD Risk Factors Changes at 12-weeks of Intervention in Obese T2D} Participants

\begin{tabular}{lllllll}
\hline \multirow{2}{*}{$\begin{array}{c}\text { Outcome } \\
\text { Measurement }\end{array}$} & \multicolumn{4}{c}{ MRT (n=12) } & \multicolumn{2}{c}{ SDT (n=13) } \\
\cline { 2 - 8 } & Baseline & \multicolumn{1}{c}{ 12-weeks } & MD (95\% CI) & Baseline & 12-weeks & \multicolumn{1}{c}{ MD (95\% CI) } \\
TC:HDL-C & $4.82 \pm 0.43$ & $4.43 \pm 0.38$ & $-0.39(0.04-0.74) *$ & $4.68 \pm 0.23$ & $4.40 \pm 0.32$ & $-0.29(-0.24-0.82)$ \\
SBP $(\mathrm{mmHg})$ & $130.00 \pm 4.45$ & $123.54 \pm 3.31$ & $-6.46(0.66-12.25) *$ & $134.19 \pm 6.04$ & $125.50 \pm 4.83$ & $-8.70(-0.73-18.12)$ \\
DBP $(\mathrm{mmHg})$ & $85.96 \pm 2.47$ & $79.58 \pm 2.22$ & $-6.38(2.35-10.40) *$ & $86.50 \pm 11.39$ & $80.73 \pm 2.89$ & $-5.77(0.32-11.22) *$ \\
VF $(\%)$ & $17.92 \pm 1.37$ & $16.67 \pm 1.16$ & $-1.25(0.59-1.90) *$ & $15.60 \pm 1.34$ & $14.85 \pm 1.41$ & $-0.75(-0.30-1.53)$ \\
BMI $\left(\mathrm{kg} / \mathrm{cm}^{2}\right)$ & $31.40 \pm 1.18$ & $30.10 \pm 1.14$ & $-1.30(0.95-1.65) *$ & $33.08 \pm 1.80$ & $32.60 \pm 1.98$ & $-0.48(-0.09-1.05)$ \\
hs-CRP $(\mathrm{mg} / \mathrm{L})^{\dagger}$ & $4.06 \pm 0.81$ & $3.27 \pm 0.66$ & $-0.79(0.18-1.40) *$ & $3.81 \pm 1.26$ & $3.22 \pm 0.97$ & $-0.60(-0.51-1.76)$
\end{tabular}
$\mathrm{MRT}=$ Meal replacement therapy, $\mathrm{SDT}=$ Standard dietetic therapy, $\mathrm{MD}=$ mean difference, $\mathrm{Cl}=$ confidence interval, $\mathrm{SEM}=$ standard error mean, $\mathrm{n}=$ number of participants, $\mathrm{TC}: \mathrm{HDL}-\mathrm{C}$
$=$ total cholesterol: high-density lipoprotein cholesterol, $\mathrm{SBP}=$ systolic blood pressure, $\mathrm{DBP}=$ diastolic blood pressure, $\mathrm{VF}=$ visceral fat, $\mathrm{BMI}=$ body mass index, hs-CRP $=$ highsensitivity $C$-reactive protein at ${ }^{*}=P<0.05$. ths-CRP participants assessed for MRT were $n=10$. Data at baseline and 12-weeks are means $\pm S E M$.

Figure 1: Clinical Phenotypes and Genes Transcription Predictors of Atherogenic Index

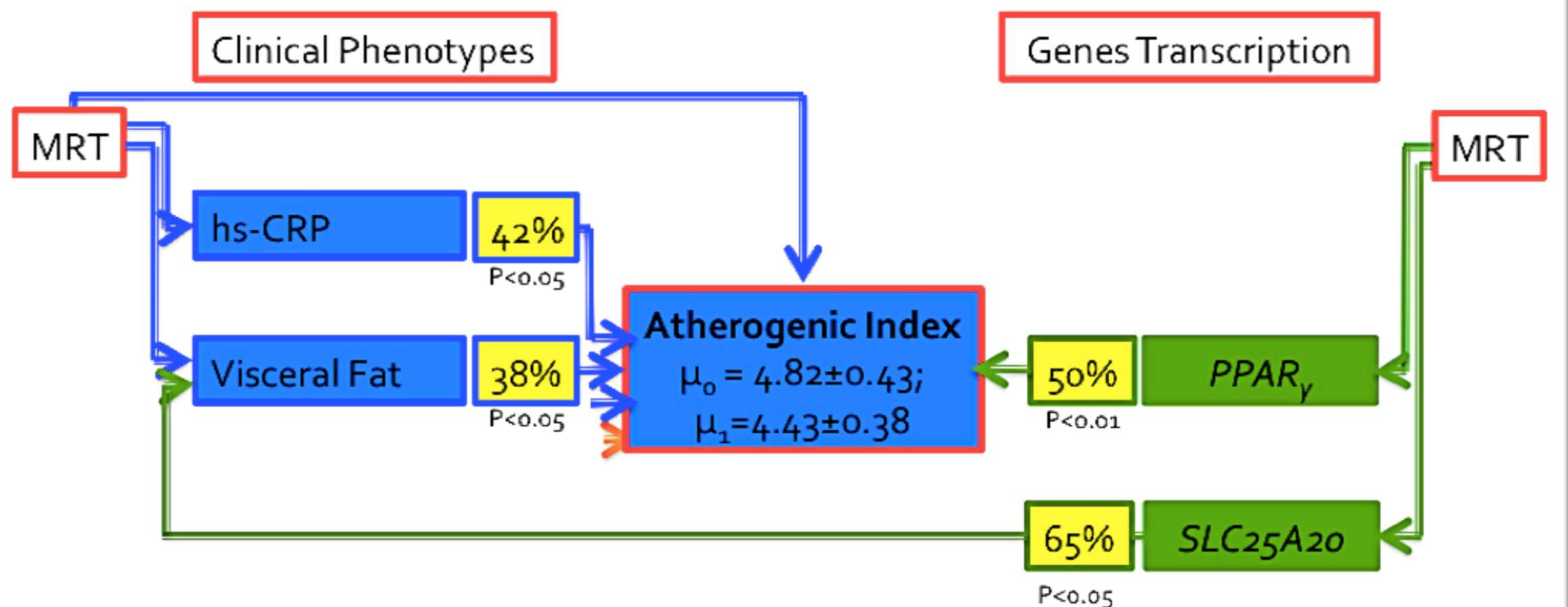

Legend:

CVD Risk Factors Reduction from baseline $(P<0.05)$ $\mathbf{R}^{2}$, Coefficient of determination
References :

1. Fox CS, Golden SH, Anderson C, Bray GA, Burke LE, de Boer IA, Deedwania P, Robert H. Eckel, Ershow AG, Fradkin J, Inzucchi SE Kosiborod M, Nelson RG, Patel MJ, Pignone M Quin Schauer PR, Selvin E, Vafiadis DK. 2015. Update on Prevention of Cardiovascular Disease in Adults With Type 2 Diabetes Mellitus in Light of Recent Evidence A Scientific Statement From the American Heart Association and the American Diabetes Association. Diab Care. 38(9): 17771803.

Wang YX. 2010. PPARs: diverse regulators in energy metabolism and metabolic diseases. Cell Research. 20:124-137.

3. Khera AV, Emdin CA, Drake I, Natarajan P, Bick AG, Cook NR, Chasman DI, Baber U, Mehran R, Rader DJ, Fuster V, Boerwinkle E, Melander O, Orho-Melander M, Ridker PM, Kathiresan S. 2016. Genetic risk, adherence to a healthy lifestyle, and coronary disease. N Eng J Med. 375:2349-358

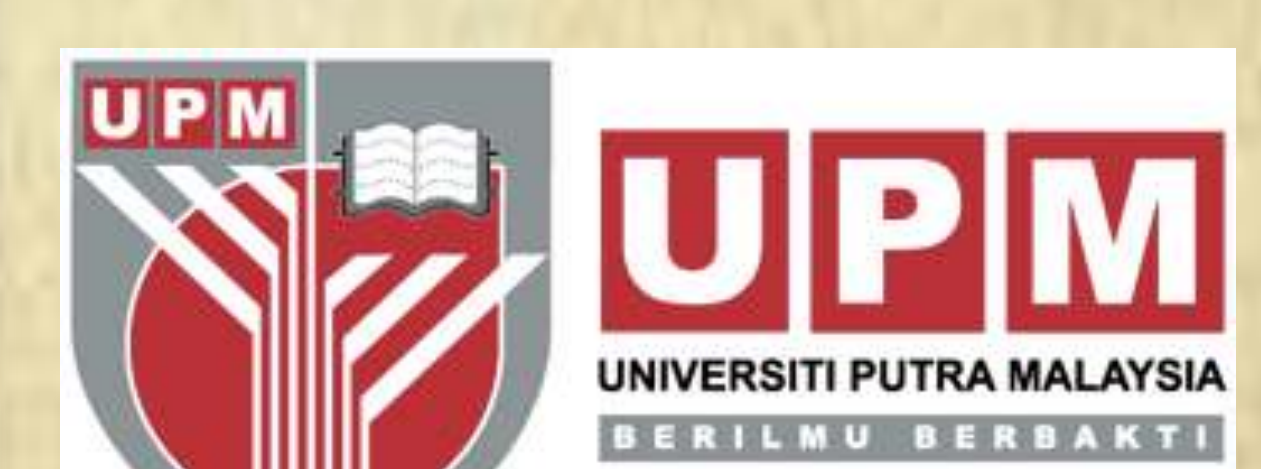

